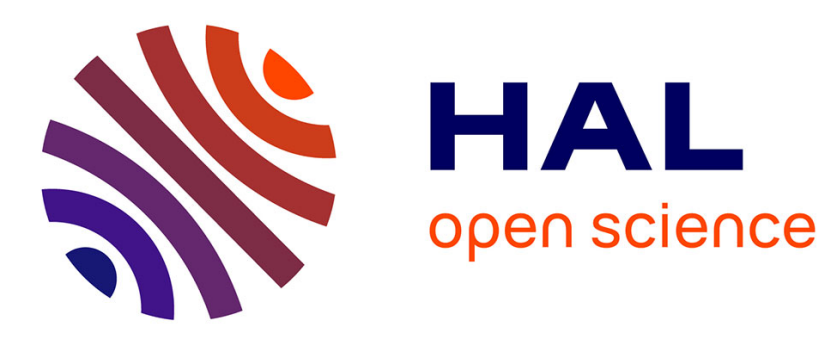

\title{
3D Walking Biped: Optimal Swing of the Arms
}

Yannick Aoustin, A. M. Formalskii

\section{To cite this version:}

Yannick Aoustin, A. M. Formalskii. 3D Walking Biped: Optimal Swing of the Arms. Multibody System Dynamics, 2013, pp.10.1007/s11044-013-9378-3. 10.1007/s11044-013-9378-3 . hal-00839761

\section{HAL Id: hal-00839761 https://hal.science/hal-00839761}

Submitted on 30 Jun 2013

HAL is a multi-disciplinary open access archive for the deposit and dissemination of scientific research documents, whether they are published or not. The documents may come from teaching and research institutions in France or abroad, or from public or private research centers.
L'archive ouverte pluridisciplinaire HAL, est destinée au dépôt et à la diffusion de documents scientifiques de niveau recherche, publiés ou non, émanant des établissements d'enseignement et de recherche français ou étrangers, des laboratoires publics ou privés. 


\title{
3D Walking Biped: Optimal Swing of the Arms
}

\author{
Y. Aoustin
}

L'UNAM, Institut de Recherche en

Communications et Cybernétique de Nantes, UMR 6597,

CNRS, École Centrale de Nantes, Université

de Nantes, France.

A. M. Formalskii

Institute of Mechanics, Lomonosov Moscow

State University.

the date of receipt and acceptance should be inserted later

\begin{abstract}
A ballistic walking gait is designed for a 3D biped with two identical twolink legs, a torso, and two identical one-link arms. In the single support phase, the biped moves due to the existence of a momentum, produced mechanically, without applying active torques in the inter-link joints. This biped is controlled with impulsive torques at the instantaneous double support to obtain a cyclic gait. The impulsive torques are applied in the seven inter-link joints. Then an infinity of solutions exists to find the impulsive torques. An effort cost functional of these impulsive torques is minimized to determine a unique solution. Numerical results show that for a given time period and a given length of the walking gait step, there is an optimal swinging amplitude of the arms. For this optimal motion of the arms, the cost functional is minimum.
\end{abstract}

Keywords: Arms, Biped, Ballistic walking gait, Impulsive torques, Optimization, Instantaneous double support.

\section{Introduction}

The motion of the arms has an effect, which is not well-known, on the human locomotion [1-3]. This influence is studied in our paper.

Currently, many papers are devoted to the behavior of the locomotor system of bipeds. However few studies and results exist to describe the influence of the trunk and the arms on the dynamic walking gaits for a 3D biped. For instance, Pontzer et al. [3], from analysis of human walking and running, support a passive arm swing hypothesis for upper body movement. In order to study the influence of the arms on elderly adults,

Corresponding author: Address: 1, rue de la Noë, BP 92101. 44321 Nantes, France. (e-mail: Yannick.Aoustin@irccyn.ec-nantes.fr).

Address: 1, Michurinskii Prospect, Moscow, 119192, Russia. (e-mail: formal@imec.msu.ru). This work was supported by Ministry of Education and Science of Russian Federation, Project 07.524.11.4012 and CNRS via a Project of International Collaboration Scientific, PICS 3866. 
Ortega et al [1] prove that the greater metabolic cost of walking in elderly adults is not caused by a greater cost of lateral stabilization. Moreover, arm swing reduces the metabolic cost of walking for both young and elderly adults, likely by contributing to stability. Bruijn et al. [4] show that pelvic and thoracic contributions to total body angular momentum is low, while contributions of legs and arms are much larger for human walking. Collins et al. [2] show that simulation results and experimental data both support the proposition that the primary function of the arms during gait is to reduce the fluctuations in vertical angular momentum defined with respect to the body center of mass and external moment requirements. Therefore arm swinging is not an evolutionary relic from quadrupedalism that serves little or no purpose but minimizes energy use in locomotion. From biomechanics observations authors such as Umberger [5] show that during walking the arms reduce a yaw moment, which acts on the sole of the stance foot. This yaw moment is due to the non-symmetric roles of the swing and the stance legs. Then for the bipedal robots the optimization of the amplitude of the arm-swing during walking can minimize the effort cost functional.

To understand and to reproduce the influence of the human's bodies during walking with a biped is a difficult task. The human walking gait is the result of a complex orchestration of muscle forces, joint motions, and neural motor commands; the energetic cost of producing muscle forces is complex to analyze, see Hill [6], Doke and Kuo [7], Vaughan [8]. In our point of view, the study of ballistic walking of biped, along with human experiments, may provide insight in human walking. If human and animal motions comprise alternating periods of muscle activity and relaxation, and the double support is relatively short, then it is logical to consider the problem of purely ballistic swing phases and double support phases with impulsive inter-link torques. Similar statement of the problem is proposed by Formal'skii [9], [10], [11], Mochon and McMahon [12], and McGeer [13]. This kind of motion with ballistic parts and impulsive torques appears to be less energy consuming, see Formal'skii [10].

We consider an anthropomorphic 3D biped with a pelvis, a torso, and one-link arms but without feet (with point feet). The contribution of this paper is to solve for this biped a boundary value problem to find a walking ballistic gait, which is cyclic, with instantaneous double supports and impulsive torques, and to study the effect of the arm-swing. Furthermore, we compute a minimum of the cost functional, which is calculated through the impulsive torques during the instantaneous double support phase. The research of these minima is done as a function of the amplitude of the arm-swing. The impulsive control torques, which are applied in the inter-link joints between the neighboring single support phases are described by delta-functions of Dirac. Of course, it is not possible to realize these impulsive control torques. Therefore, our approach can be considered as asymptotic. Using this approach we can evaluate the role of the arm-swing in the walking process.

The rest of the paper is outlined as follows. Section 2 is devoted to the biped model presentation. Problem definition of the ballistic walking is given in Section 3. In the same Section 3, the algebraic equations for the instantaneous double support are designed. The effort cost functional for the impulsive control is presented in Section 4. The results of simulation are shown in Section 5. Our conclusion and perspectives are offered in Section 6. 
2 Biped Model

2.1 Physical parameters of the biped

For the seven-link biped (Figure 1) we use the physical parameters from [10]. The

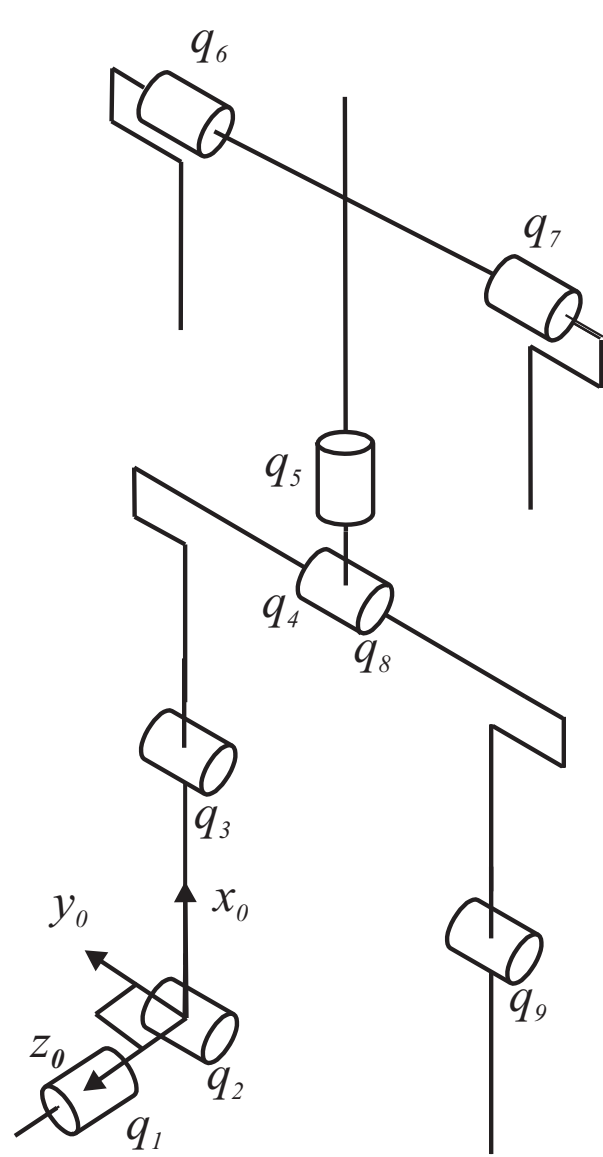

(a)

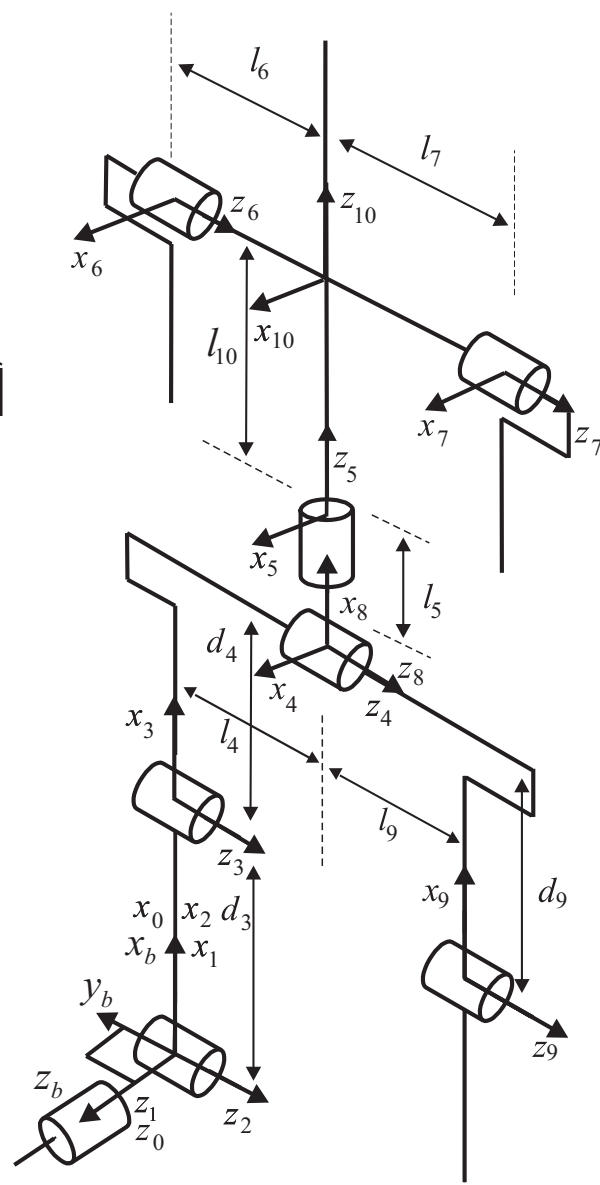

(b)

Fig. 1 The kinematic model with its DOF and link frames of the biped.

whole mass of the biped is $75 \mathrm{~kg}$, its height is $1.75 \mathrm{~m}$. Figure 2 the distance between the knee joint and the center of mass for the shin is: $S_{s}=0.324 \mathrm{~m}$, between the hip joint and the center of mass for the thigh: $S_{t}=0.18 \mathrm{~m}$, between the hip joint and the center of mass for the trunk: $S_{T}=0.386 \mathrm{~m}$, and between the shoulder joint and the center of mass for the arm: $S_{a}=0.33 \mathrm{~m}$. The head mass is included in the trunk that its length is $l_{T}$. The distance between the shoulder joints and the hip joints is: $l_{5}+l_{10}=S_{b}=0.5 \mathrm{~m}$. Table 2.1 gathers the masses, the lengths and the inertia moments for each link of the biped. 


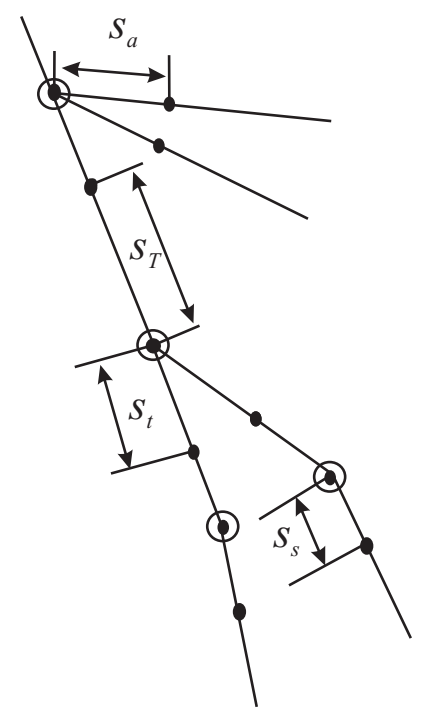

Fig. 2 Schematic in the sagittal plane of the biped with the position of the centre of mass of each link.

\subsection{Geometric description of the biped}

The studied anthropomorphic biped is depicted in Figure 1. All bodies (links) are supposed massive, rigid, and are connected by revolute joints. The biped has a pelvis, a torso, two identical one-link arms, and two identical two-link legs with one degree-offreedom revolute knees that are terminated with "point" feet. Each hip and shoulder contains a revolute joint with one degree of freedom. The torso has a one degreeof-freedom revolute joint. This 3D biped is represented as a tree structure. In single support, the stance leg tip is assumed to have no sliding motion, no take-off and no yaw motion. Then the stance leg tip acts as a passive pivot in the sagittal and frontal planes, and is modeled as a foot with two unactuated degrees of freedom. The friction ground reaction is assumed sufficiently large to avoid a sliding sole around the vertical axis. This model corresponds to the case of a biped with feet when the size of the feet decreases to zero. The stance leg tip is the base of the tree structure.

Remark. During the rectilinear human walking the yaw motion in the ankle joint of the stance leg is small. The yaw motion of the biped is introduced by the yaw motion of the torso.

In Figure 1(a) the position of frames is defined according to the Denavit-Hartenberg convention. The vector of the nine generalized coordinates is $\mathbf{q}=\left[q_{1}, q_{2}, q_{3}, q_{4}, q_{5}, q_{6}, q_{7}, q_{8}, q_{9}\right]^{\mathrm{t}}$. The absolute angles $q_{1}$ and $q_{2}$ are respectively the roll and pitch angles of the stance leg, Figure 1(a). The variables $q_{3}$ and $q_{9}$ describe the relative joint angles of the stanceleg and swing-leg knees, respectively. The variable angle $q_{4}$ is the joint angle of the stance leg hip relative to the pelvis. The variable angle $q_{5}$ is the joint angle to describe the yaw motion of the torso relatively to the pelvis. The variable angle $q_{8}$ is the joint angle of the swing leg hip relative to the pelvis. Variables $q_{6}$ and $q_{7}$ describe the joint angles of the two arms with respect to the torso. The joint angles $q_{1}$ and $q_{2}$ are unac- 


\begin{tabular}{|c|c|c|c|c|}
\hline & Thigh & Shin & Trunk & Arm \\
\hline Length $(m)$ & $l_{t}=d_{4}=0.41$ & $l_{s}=d_{3}=0.497$ & $l_{T}=0.625$ & $l_{a}=0.66$ \\
\hline Mass $(k g)$ & $m_{t}=8.6$ & $m_{s}=4.6$ & $m_{T}=16.5$ & $m_{a}=4.6$ \\
\hline $\begin{array}{c}\text { Inertia in } x \\
\left(\mathrm{~kg} \cdot \mathrm{m}^{2}\right)\end{array}$ & $I_{x}^{t}=0$ & $I_{x}^{s}=0$ & $I_{x}^{T}=11.3$ & $I_{x}^{a}=0$ \\
\hline $\begin{array}{c}\text { Inertia in } y \\
\left(\mathrm{~kg} \cdot \mathrm{m}^{2}\right)\end{array}$ & $I_{y}^{t}=0.7414$ & $I_{y}^{s}=0.0521$ & $I_{y}^{T}=11.3$ & $I_{y}^{a}=0.7414$ \\
\hline $\begin{array}{c}\text { Inertia in } z \\
\left(\mathrm{~kg} \cdot \mathrm{m}^{2}\right)\end{array}$ & $I_{z}^{t}=0.7414$ & $I_{z}^{s}=0.0521$ & $I_{z}^{T}=11.3$ & $I_{z}^{a}=0.7414$ \\
\hline $\begin{array}{c}\text { Hip } \\
\text { distances }(m)\end{array}$ & \multicolumn{4}{|c|}{$l_{4}=l_{9}=0.20$} \\
\hline $\begin{array}{c}\text { Shoulder } \\
\text { distances }(m)\end{array}$ & \multicolumn{4}{|c|}{$l_{6}=l_{7}=0.22$} \\
\hline $\begin{array}{c}\text { distance } \\
\text { Shoulders/Torso } \\
\text { Joint }(m) \\
\end{array}$ & \multicolumn{4}{|c|}{$l_{10}=0.5$} \\
\hline $\begin{array}{l}\text { Torso Joint } \\
\text { /Pelvis }(m)\end{array}$ & \multicolumn{4}{|c|}{$l_{5}=0.0$} \\
\hline
\end{tabular}

Table 1 Biped's Parameters

tuated, due to the passive contact, while $q_{3}, q_{4}, q_{5}, q_{6}, q_{7}, q_{8}$, and $q_{9}$ are independently actuated.

2.3 Equations of motion of the biped in swing phase

The equations of motion of the $3 D$ seven-link biped in the swing phase are obtained through the Newton-Euler method, and they have the following symbolical form,

$$
\left[\begin{array}{c}
\mathbf{F}_{0} \\
\boldsymbol{\Gamma}
\end{array}\right]=\mathrm{NE}(\mathbf{q}, \dot{\mathbf{q}}, \ddot{\mathbf{q}})
$$

where $\boldsymbol{\Gamma}$ is the $7 \times 1$ joint torques vector, $\mathbf{F}_{0}$ is a $4 \times 1$ vector composed of the three components of the ground force and one moment around the vertical axis on the stance leg tip. The number of degrees of freedom is nine, but there are only seven torques. This means that the biped is an under actuated mechanism during the single support phase.

Remark:

- At the impact, the previous supporting foot becomes the swing foot, and its velocity after impact can differ from zero. Consequently, the modeling of the biped must be able to describe a non-fixed stance leg tip. Since the dynamic model is calculated 
with the Newton-Euler algorithm, it is convenient to define the linear velocity of the origin of frame $R_{0}$ with the Newton variables. This origin coincides with the stance leg tip. Furthermore, the biped's hips have a single revolute joint. There are no adduction-abduction movements and internal-external rotation. Then for the impact model, or the double support model the biped's position with respect to an inertial frame can be expressed by $\mathbf{X}=\left[x_{0}, y_{0}, z_{0}, \mathbf{q}^{\mathrm{t}}\right]^{\mathrm{t}}$, where $x_{0}, y_{0}$, and $z_{0}$ are the Cartesian coordinates of the origin of frame $R_{0}$.

\subsection{Double Support}

During the biped's gait, an impact occurs at the end of a single support phase, when the swing leg tip touches the ground. At the instant of impact, denoted by $T$, the double support phase is assumed instantaneous. At the instant of the passive inelastic impact, the biped looses energy. Therefore, the velocity vector after the impact will not be the desired one, if the bearing surface is horizontal. Then for the next ballistic step the desired initial velocity vector will not be reached. As a consequence, a complete walking cyclic gait of the biped cannot be realized on a horizontal surface without active torques. However, theoretically, around the instantaneous double support it is necessary to define impulsive torques in order to ensure the desired velocity jump, see Formalskii [10], [11], Hurmuzlu, and Chang [14]. In the next Section, it is shown how to define these impulsive torques.

\section{Ballistic motion and impulsive control: Problem definition}

\subsection{Single support}

In the single support phase, the stance leg tip is assumed to act as a passive pivot in the sagittal and frontal planes with no yaw motion, no sliding motion, no take-off. In this case, it is possible to model the biped as a manipulator robot with a tree structure and nine degrees of freedom. The Lagrangian dynamic model of the biped in the swing motion can be presented under the form of the following matrix equation

$$
\mathbf{D}(\mathbf{q}) \ddot{\mathbf{q}}+\mathbf{C}(\mathbf{q}, \dot{\mathbf{q}})+\mathbf{G}(\mathbf{q})=\mathbf{B} \Gamma .
$$

Here $\mathbf{D}(\mathbf{q})$ is the symmetric, positive definite $9 \times 9$ inertia matrix; $\mathbf{C}(\mathbf{q}, \dot{\mathbf{q}})$ and $\mathbf{G}(\mathbf{q})$ are the $9 \times 1$ vectors of the centrifugal, Coriolis and gravity forces respectively. The $9 \times 7$ matrix $\mathbf{B}=\left[\mathbf{0}_{7 \times 2}, \mathbf{I}_{7 \times 7}\right]^{\mathrm{t}}$ is used to indicate whether a joint is actuated or not. The notations $\mathbf{0}_{7 \times 2}$ and $\mathbf{I}_{7 \times 7}$ define the zero $7 \times 2$ matrix and the $7 \times 7$ identity matrix, respectively. All matrices and vectors $\mathbf{D}, \mathbf{C}$, and $\mathbf{G}$ are calculated through the Newton Euler equations.

Let $\mathbf{q}(0)$ be the initial configuration of the biped at time $t=0$. We assume that in the sagittal plane, the front and hind legs are the stance and swing legs respectively. The final configuration of the biped in the single support phase at the given time $t=T$ is noted $\mathbf{q}(T)$. Let this given configuration be similar to the initial configuration with the legs swapped. Let $L$ be the length of the step corresponding to a single support. We consider a ballistic motion during the single support phase with $\boldsymbol{\Gamma}=\mathbf{0}_{7 \times 1}$. Consequently, the equation (2) for ballistic motion becomes:

$$
\mathbf{D}(\mathbf{q}) \ddot{\mathbf{q}}+\mathbf{C}(\mathbf{q}, \dot{\mathbf{q}})+\mathbf{G}(\mathbf{q})=\mathbf{0}_{9 \times 1} .
$$


To design the ballistic walking, it is necessary to find the solution $\mathbf{q}(t)$ of the matrix equations (3) with the given boundary conditions $\mathbf{q}(0)$ and $\mathbf{q}(T)$. We have to find the initial velocity vector $\dot{\mathbf{q}}(0)$ such that solution $\mathbf{q}(t)$, starting from the given initial configuration $\mathbf{q}(0)$ with the velocity vector $\dot{\mathbf{q}}(0)$, reaches the given final configuration $\mathbf{q}(T)$ at the given time $T$. The given boundary conditions $\mathbf{q}(0)$ and $\mathbf{q}(T)$ are chosen such that the positions of the locomotor system and the trunk of the biped are similar to human configurations. This boundary value problem can be numerically solved using a Newton method with vector $\dot{\mathbf{q}}(0)$ unknown. The motion of the biped is admissible, if the vertical component of the ground reaction in the stance leg is positive (directed upwards), and if the swing leg moves over the ground for $0<t<T$. We check these constraints after solving the boundary value problem - a posteriori. The ground reaction is calculated from (1) using the forward and the backward recursions.

After solving the boundary value problem, the vector of the initial velocities $\dot{\mathbf{q}}(0)$ is known. We denote it by $\dot{\mathbf{q}}^{a}$. If the initial conditions $\mathbf{q}(0), \dot{\mathbf{q}}^{a}$ are known, then by integration of the system (3) the vector of the terminal velocities $\dot{\mathbf{q}}(T)$ can also be found. We denote it by $\dot{\mathbf{q}}^{b}$. The stance point foot is defined as the origin point of frame $R_{0}$ during the ballistic motion. Then in order to write the impulsive impact equations for the initial time and the final time of the ballistic motion, we introduce the extended

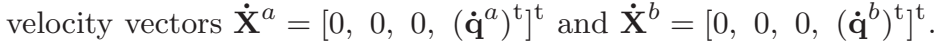

\subsection{Structure of double support phase}

The Lagrangian dynamic model of the swing motion, which takes into account the $3 \times 1$ ground reaction $\mathbf{R}_{j}$ for the supporting leg $j$ ( $j=1$ or 2 ) can be presented in the following matrix form:

$$
\mathbf{D}_{e} \ddot{\mathbf{X}}+\mathbf{C}_{e}(\mathbf{q}, \dot{\mathbf{q}})+\mathbf{G}_{e}(\mathbf{q})=\mathbf{B}_{e} \boldsymbol{\Gamma}+\mathbf{J}_{j}^{\mathrm{t}} \mathbf{R}_{j}
$$

Here $\mathbf{D}_{e}(\mathbf{q})$ is the symmetric, positive definite $12 \times 12$ inertia matrix; $\mathbf{C}_{e}(\mathbf{q}, \dot{\mathbf{q}})$ and $\mathbf{G}_{e}(q)$ are the $12 \times 1$ vectors of the centrifugal, Coriolis and gravity forces respectively; the $12 \times 7$ matrix $\mathbf{B}_{e}=\left[\mathbf{0}_{7 \times 5}, \mathbf{I}_{7 \times 7}\right]^{\mathrm{t}}$ is used to indicate whether a joint is actuated or not; $\mathbf{J}_{j}(\mathbf{q})(j=1,2)$ are the $3 \times 12$ Jacobian matrices of constraint functions. The Jacobian matrix $\mathbf{J}_{j}(\mathbf{q})$ can be calculated with the backward recursion of the algorithm of Newton-Euler, by considering the velocity and acceleration of each link as null. These constraints are relative to the contact between the ground and the tip of the stance leg $j$. Setting to zero the acceleration of the stance leg tip

$$
\mathbf{J}_{j}(\mathbf{q}) \ddot{\mathbf{X}}+\mathbf{H}_{j}(\mathbf{q}, \dot{\mathbf{q}})=0
$$

implies that the Cartesian coordinates of the leg tip $j$ do not change, if its initial velocity is null. $\mathbf{H}_{j}(\mathbf{q}, \dot{\mathbf{q}})=\dot{\mathbf{J}}_{j} \dot{\mathbf{X}}$ is a $3 \times 1$ vector.

Let us consider the current ballistic motion on the stance leg 1 and the following ballistic motion on the stance leg 2 . Let the final velocity vector $\dot{\mathbf{X}}^{b}$ of the current ballistic swing motion and the initial velocity vector $\dot{\mathbf{X}}^{a}$ of the next ballistic swing motion be known from the solution of the boundary value problem ${ }^{1}$ and the numerical integration of the matrix equation (3) or (4) with $\Gamma=0, \ddot{x}_{0}=\ddot{y}_{0}=\ddot{z}_{0}=0$. Let us apply the impulsive torques in the joints with the intensity vectors $\mathbf{I}^{-}$and $\mathbf{I}^{+}$,

1 Therefore there is a permutation operation between $\dot{\mathbf{X}}^{a}$ and the solution of the boundary value problem to take into account the exchange of the role of both legs. 
respectively just before and just after the passive impact with the ground to create a complete cyclic motion. Then we divide the instantaneous double support phase into three sub-phases and detail these sub-phases, which are presented in Figure 3.

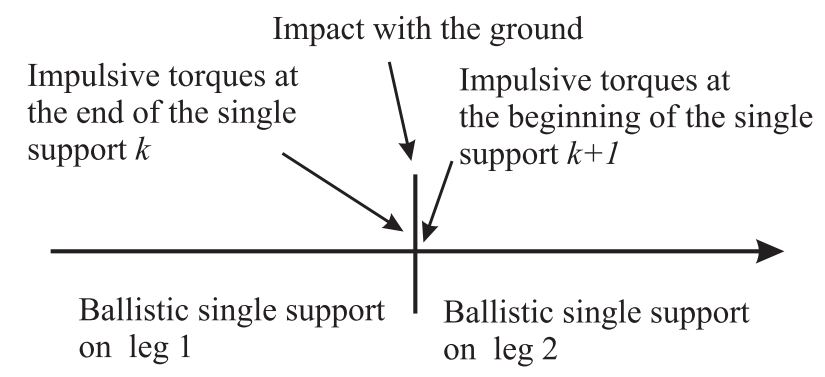

Fig. 3 Decomposition of the impulsive impact.

- The swing leg 2 touches the ground at the end of the ballistic single support motion on leg 1, and an impact occurs. Just before contact with the ground, in the first subphase at time $T^{-}$, impulsive torques $\boldsymbol{\Gamma}^{-}(t)=\mathbf{I}^{-} \delta\left(t-T^{-}\right)$are applied at the seven inter-link joints. Here $\delta\left(t-T^{-}\right)$is the Dirac delta-function. At the same instant $T^{-}$, the impulsive ground reaction $\mathbf{R}_{1}^{-}=\mathbf{I}_{\mathbf{R}_{1}}^{-} \delta\left(t-T^{-}\right)$is applied in the hind leg tip. Here $\mathbf{I}_{\mathbf{R}_{1}}^{-}\left(I_{R_{1 x}}^{-}, I_{R_{1 y}}^{-}, I_{R_{1 z}}^{-}\right)$is the vector of the magnitudes of the impulsive reaction in leg 1 . Under the impulsive torques, the velocity vector $\dot{\mathbf{X}}$ of the biped changes instantaneously from the value $\dot{\mathbf{X}}^{b}$ to some value $\dot{\mathbf{X}}^{-}$. The corresponding equations for the velocities jump can be obtained through the integration of equations of motion (4), (5) for the infinitesimal time from $T^{-}$to $T$. The torques provided by the Coriolis and gravity forces have finite values. Thus, they do not influence the velocity jump:

$$
\begin{gathered}
\mathbf{D}_{e}[\mathbf{q}(T)]\left(\dot{\mathbf{X}}^{-}-\dot{\mathbf{X}}^{b}\right)=\mathbf{B}_{e} \mathbf{I}^{-}+\mathbf{J}_{1}^{\mathrm{t}}[\mathbf{q}(T)] \mathbf{I}_{R_{1}}^{-} \\
\mathbf{J}_{1}[\mathbf{q}(T)]\left(\dot{\mathbf{X}}^{-}-\dot{\mathbf{X}}^{b}\right)=\mathbf{0}_{3 \times 1}
\end{gathered}
$$

Here $\mathbf{q}(T)$ denotes the configuration of the biped at the instant of impulsive actions (at the double support). This configuration does not change at the instants of the first, second, and third sub-phases. The velocity of the hind leg tip remains zero after the first sub-phase. Then the biped has the velocity vec-

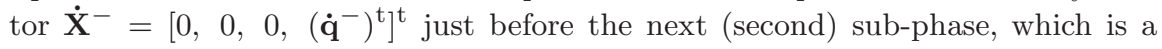
passive impact with the ground.

- The second sub-phase is assumed to be a passive impact, i.e. without torques applied in the inter-link joints, absolutely inelastic, and such that the legs do not slip. Given these conditions, the ground reactions at the instant of an impact can be considered as impulsive forces and defined by the delta-functions $\mathbf{R}_{2}=\mathbf{I}_{\mathbf{R}_{2}} \delta(t-$ $T)$. Here $\mathbf{I}_{\mathbf{R}_{2}}\left(I_{R_{2 x}}, I_{R_{2 y}}, I_{R_{2 z}}\right)$ is the vector of the magnitudes of the impulsive reaction in leg 2 , see [10]. The corresponding equations for the velocities jump can be obtained through the integration of the matrix equation (4) for the infinitesimal time. The velocity of the stance leg tip 1 before an impact is equal to zero. 
Generally speaking, two results are possible after the passive impact, if we assume that there is no slipping of the leg tips. The stance leg lifts off the ground or both legs remain on the ground. Numerical investigations were carried out after impact to check the ground reaction in the stance leg tip and the linear velocity of this leg tip. We considered numerically both cases. From these numerical investigations we concluded that the first case (stance leg lifts off the ground) takes place in all our variants. In this case, the vertical component of the velocity of the taking-off leg tip just after the impact is directed upwards. Also there is no interaction (no friction, no sticking) between the taking-off leg tip and the ground. The ground reaction in this taking-off leg tip is null. If we assume that after the impact the stance leg remains on the ground (second case), the vertical component of the ground reaction in this leg must be null or directed upwards. But our calculations show that this component is directed downwards. This means that both legs cannot remain on the ground. For the first case, the impact equations can be written in the following matrix form:

$$
\mathbf{D}_{e}[\mathbf{q}(T)]\left(\dot{\mathbf{X}}^{+}-\dot{\mathbf{X}}^{-}\right)=\mathbf{J}_{2}^{\mathrm{t}}[\mathbf{q}(T)] \mathbf{I}_{\mathbf{R}_{2}}
$$

Here $\dot{\mathbf{X}}^{+}$is the velocity vector just after an inelastic passive impact. The swing leg 2 after the impact becomes a stance leg. Therefore, its tip velocity becomes zero after the impact,

$$
\mathbf{J}_{2}[\mathbf{q}(T)] \dot{\mathbf{X}}^{+}=\mathbf{0}_{3 \times 1}
$$

- The swing leg 1 takes off the ground at the second sub-phase, which is the passive impact. Then, the next ballistic single support motion on leg 2 starts. However, before the next ballistic swing motion (just after the take off), in the third sub-phase at time $T^{+}$, impulsive torques $\boldsymbol{\Gamma}^{+}(t)=\mathbf{I}^{+} \delta\left(t-T^{+}\right)$are applied in the inter-link joints to change the velocity of the biped instantaneously from the velocity vector just after passive impact $\dot{\mathbf{X}}^{+}$to the known velocity vector $\dot{\mathbf{X}}^{a}$. Integrating the differential equations (4) we come to the following matrix relation:

$$
\mathbf{D}_{e}[\mathbf{q}(T)]\left(\dot{\mathbf{X}}^{a}-\dot{\mathbf{X}}^{+}\right)=\mathbf{B}_{e} \mathbf{I}^{+}+\mathbf{J}_{2}^{\mathrm{t}}[\mathbf{q}(T)] \mathbf{I}_{\mathbf{R}_{2}}^{+}
$$

System (6)-(10) is composed of 42 scalar equations to find 47 unknown variables, which are the components of the vectors: $\dot{\mathbf{X}}^{-}(12 \times 1), \mathbf{I}^{-}(7 \times 1), \mathbf{I}_{\mathbf{R}_{1}}^{-}(3 \times 1)$ (for the first sub-phase), $\dot{\mathbf{X}}^{+}(12 \times 1), \mathbf{I}_{\mathbf{R}_{2}}(3 \times 1)$ (for the second sub-phase), $\mathbf{I}^{+}(7 \times 1)$ and $\mathbf{I}_{\mathbf{R}_{2}}^{+}(3 \times 1)$ (for the third sub-phase). Then the problem of impulsive control has an infinite number of solutions. But if the number of equations is less than the number of unknown variables, it is possible to extract a unique solution minimizing some cost functional. The components of the above-mentioned vectors are the subjects of the minimization. Among this set of components, five can be defined as parameters to minimize a cost functional.

\section{Effort cost functional of impulsive control}

The choice of a cost functional for a walking biped is complex. Assumptions can be stated like the actuators are not regenerative - energy cannot be restored in the muscles. Then the cost functional can be defined from the energy consumption similarly to Channon et al. [15] and Formal'skii [10] or from the torques, see Chevallereau and Aoustin [16]. We do not know if a cost functional is optimized during a human walking, 
and if yes, which cost functional. To try to introduce the muscle effects, we have chosen the following effort cost functional:

$$
W=\sum_{i=1}^{7}\left[\int_{T^{-}}^{T}\left|\Gamma_{i}^{-}(t)\right| \mathrm{d} t+\int_{T}^{T^{+}}\left|\Gamma_{i}^{+}(t)\right| \mathrm{d} t\right]
$$

But $\boldsymbol{\Gamma}^{-}(t)=\mathbf{I}^{-} \delta\left(t-T^{-}\right)$and $\boldsymbol{\Gamma}^{+}(t)=\mathbf{I}^{+} \delta\left(t-T^{+}\right)$. Here $\delta(t)$ means delta-function. Then after calculating the integrals in expression (11) the effort cost functional becomes:

$$
W=\sum_{i=1}^{7}\left[\left|I_{i}^{-}\right|+\left|I_{i}^{+}\right|\right]
$$

In simulation, with a given length $L$ and a time period $T$ of the step, for each arms amplitude, we choose a unique solution of the system (6) - (10) by minimizing quantity (12). We take into account the following constraints: $I_{R_{1 x}}^{-}>0, I_{R_{2 x}}>0$, and $I_{R_{2 x}}^{+}>0$. Furthermore, we ensure that the linear vector of the swing leg tip just before the passive impact is directed to the ground and just after the passive impact is directed to the top. Therefore, our minimization problem is the problem of parametric minimization with constraints. We used the $S Q P$ method (Sequential Quadratic Programming) [17], [18] with the fmincon function of Matlab $\AA$ to solve this problem numerically.

We consider the minimal quantity (12) corresponding to this solution as the effort cost functional for the biped walking with given $L, T$, and arms amplitude.

\section{Simulation}

During the walking, the swing leg moves over the support and bends with knee forward. The stance leg remains almost straight (the oscillation of the stance leg knee angle $q_{3}$ is less than $0.4^{\circ}$ ). The ballistic motion is valid because the vertical component of the ground reaction in the stance leg is always positive (directed upwards). These features have not been prescribed in the statement of the problem previously. There is one oscillation in the yaw joint of the trunk (variable $q_{5}$ ); the amplitude of this oscillation is close to $1.0^{\circ}$. There is a little rotation described by the roll angle $q_{1}$ of the biped in its frontal plane.

For several values of the step length $L$ and of the time period $T$, we numerically studied the influence of the arm swinging amplitude on the effort cost of the biped walking.

In Figure 4, the effort cost functional as a function of the amplitude of the armswinging is shown for the fixed time period $T=0.45 \mathrm{~s}$ and a varying step length $L$ $(L=0.40 \mathrm{~m}, 0.45 \mathrm{~m}$ and $0.50 \mathrm{~m})$. We can see from Figure 4 that for $T=0.45 \mathrm{~s}$ and $L=0.45 \mathrm{~m}$ with amplitude $23.5^{\circ}$, the effort cost functional is minimal. Consequently, this amplitude is optimal for this particular time period and the step length. These numerical results enhance the observations of experiments of Collins [2] that arm swinging minimizes energy use in locomotion. This amplitude of the optimal swing of the arms is close to the human data that we can find in [3]. If the length of the step increases, the effort cost functional increases; the optimal amplitude of the arm-swing also increases. The walking of a planar biped with arms is studied by Aoustin and Formal'skii [21]. The physical parameters of the biped are the same as considered here. 


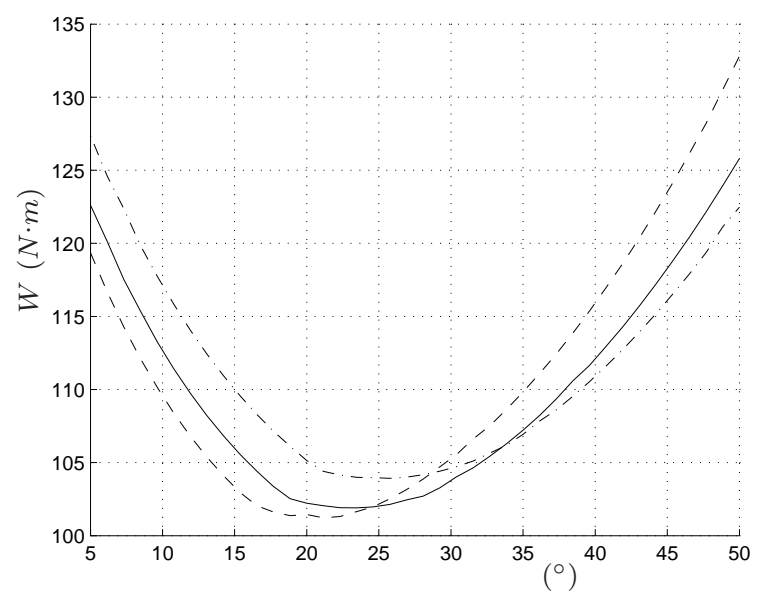

Fig. 4 Normal-gait: for $T=0.45 \mathrm{~s}$, cost functional $W$ versus motion amplitude of the arms [degrees] $L=0.40 \mathrm{~m}$ (dashed), $L=0.45 \mathrm{~m}$ (solid), and $L=0.50 \mathrm{~m}$ (dashdot).

For the case $L=0.45 \mathrm{~m}, T=0.45 \mathrm{~s}$, the optimal arms swinging amplitude found is $35.2^{\circ}$. It seems that value $23.5^{\circ}$, which is obtained here, is closer to the arm amplitude for the real human walking, see Collins et al. [2]. In literature, see Pontzer et al. [3] for human walking the observed value of the amplitude of the arms swinging is close to $20^{\circ}$.

We have compared the values of the effort cost criterion $W$ for the normal gait (like in human gait - in opposite phase) and the anti-normal gait (each arm swings in phase with the ipsilateral leg). For the case $L=0.40 \mathrm{~s}, T=0.45 \mathrm{~s}$ the optimal amplitude of the arms for the normal gait is $21.1^{\circ}$ and $W=101.22 N \cdot m$. But in this case for the same amplitude of the arms and for the anti-normal gait $W=106.07 \mathrm{~N} \cdot \mathrm{m}$. For the case $L=0.45 \mathrm{~m}, T=0.45 \mathrm{~s}$ the optimal amplitude of the arms for the normal gait equals $23.5^{\circ}$, and $W=101.9 \mathrm{~N} \cdot \mathrm{m}$. But in this case for the same amplitude of the arms and for the anti-normal gait $W=111.5 \mathrm{~N} \cdot \mathrm{m}$. For the case $L=0.50 \mathrm{~m}, T=0.45 \mathrm{~s}$ the optimal amplitude of the arms for the normal gait is $25.76^{\circ}$ and $W=103.92 \mathrm{~N} \cdot \mathrm{m}$. But in this case for the same amplitude of the arms and for the anti-normal gait $W=117.9 \mathrm{~N} \cdot \mathrm{m}$. So, for these three cases the effort cost criterion $W$ is less for the normal-gait. The different step lengths and time periods $T=0.48 \mathrm{~s}$, and $T=0.50 \mathrm{~s}$ were considered. In all considered cases, the effort cost criterion $W$ is greater for the anti-normal gaits.

In Figure 5, with the parameters $L=0.45 \mathrm{~m}, T=0.45 \mathrm{~s}$, and the amplitude of the arms equals $23.5^{\circ}$, for two successive steps the behavior of the angular momentum around the vertical axes crossed the stance point feet is drawn. The angular momentum is shown for the normal gait (see the solid line) and for the anti-normal gait (see the dashed line). Remind that at the instant of the double support the stance leg becomes transferring one, and the transferring leg becomes stance one. Therefore, the angular momentum is calculated for the first step around one vertical axis, and for the next step around another vertical axis. These successive steps are similar (symmetrical), therefore these two curves are symmetrical with respect to point $(0.45,0)$. For the Normal gait the angular momentum, at the instant $T^{+}$- just after to touch 


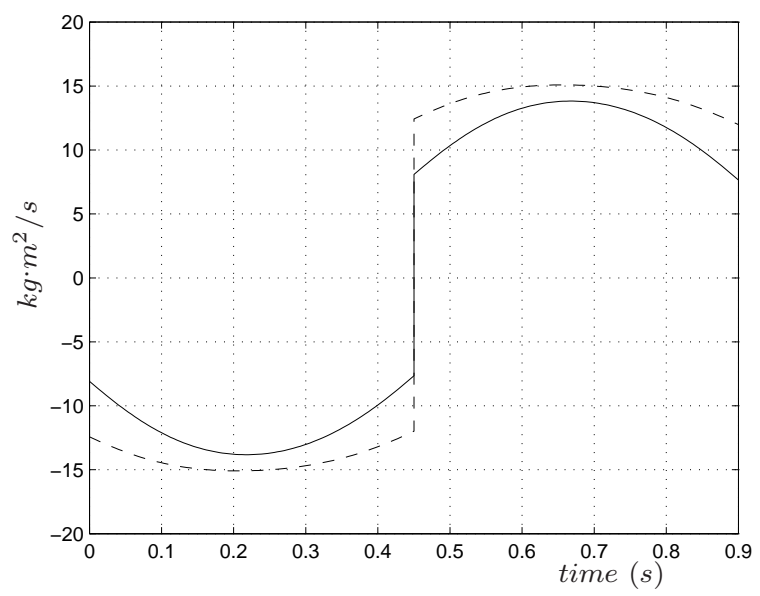

Fig. 5 For two successive steps, profiles of the angular momentum with respect to the vertical axes crossed the stance point feet, in Normal gait (solid line) and Anti-Normal gait (dashed line).

the ground, is equal to $7.65 \mathrm{~kg} \cdot \mathrm{m}^{2} / \mathrm{s}$ and to $11.98 \mathrm{~kg} \cdot \mathrm{m}^{2} / \mathrm{s}$ for the Anti-normal gait. We have calculated also the angular momentum of the biped around the vertical axis crossed the tip of the transferred leg at the instant $T^{-}$- just before to touch the ground. This angular momentum is equal to $-11.94 \mathrm{~kg} \cdot \mathrm{m}^{2} / \mathrm{s}$ for the Normal gait and to $-16.04 \mathrm{~kg} \cdot \mathrm{m}^{2} / \mathrm{s}$ for the Anti-Normal gait. So, the jump of the angular momentum for the Normal gait at the instant of double support is equal to the difference $7.65 \mathrm{~kg} \cdot \mathrm{m}^{2} / \mathrm{s}-\left(-11.94 \mathrm{~kg} \cdot \mathrm{m}^{2} / \mathrm{s}\right)=19.59 \mathrm{~kg} \cdot \mathrm{m}^{2} / \mathrm{s}$. For the Anti-normal gait, this jump equals $11.98 \mathrm{~kg} \cdot \mathrm{m}^{2} / \mathrm{s}-\left(-16.04 \mathrm{~kg} \cdot \mathrm{m}^{2} / \mathrm{s}\right)=28.02 \mathrm{~kg} \cdot \mathrm{m}^{2} / \mathrm{s}$. Thus, the jump of the angular momentum for the Normal gait is essentially less than the jump of the angular momentum for the Anti-normal gait. This means that the yaw torque in the double support, which is produced by the ground reaction torque at the supporting leg tip, and by the ground force at the other leg tip, is essentially less for the Normal gait than for the Anti-normal one. We observe this result with several ballistic walking gaits. But this difference of jump of the angular momentum, between the anti-normal mode and the normal mode for the arm swing, is less when the amplitude of this arm swing decreases.

The described results about optimal amplitude of the arm-swing and the behavior of the angular momentum complete the studies of Collins et al [2], [1], Bruijin et al [4], Eke-Okoro et al [22], and Humberger [5]. The arms mainly have a role of manipulators in daily life. But in the free balancing motion (without payload) they adopt the optimal amplitude. This amplitude increases with the velocity of the biped to balance the dynamic effects of the swing leg and thereby to reduce the effort cost functional. 


\section{Conclusion}

Using ballistic trajectories and an impulsive control, numerically we have shown the existence of an optimal amplitude of the arms swinging for a 3D biped walking. The optimal amplitudes of the arms swinging lie between 20 and 26 degrees. For three walking gaits this optimal amplitude increases with their velocity. The effort cost functional for the walk is minimal, if the arms swing with optimal amplitude. For the gait with motion of the arms and legs in phase, the effort cost functional is higher than for the gait with motion of the arms and legs in opposite phase (normal, like human gait). Our perspectives are to add a joint in each hip, and to design ballistic walking to follow curved trajectories, such as circles. The justification of the effort cost functional is still an open problem, which have to be an open future research topic to improve our understanding of the human walking gait.

\section{References}

1. J. D. Ortega, L. A. Fehlman, and C. T. Farley, "Effects of aging and arm swing on the metabolic cost of stability in human walking," Journal of Biomechanics, vol. 41, pp. 33033308, 2008.

2. S. H. Collins, P. G. Adamczyk, and A. D. Kuo, "Dynamic arm swinging in human walking," Proc. R. Soc. B (Biological Sciences), vol. 276, pp. 3679-3688, doi:10.1098/rspb.2009.0664, 2009.

3. H. Pontzer, J. H. Holloway, D. A. Raichlen, and D. E. Lieberman, "Control and function of arm swing in human walking and running," The Journal of Experimental Biology, vol. 212, pp. 523-534, 2009.

4. S. M. Bruijn, O. G. Meijer, J. H. van Dieën, I. Kingma, and C. J. C. Lamoth, "Coordination of leg swing, thorax rotations, and pelvis rotations during gait: The organisation of total body angular momentum," Gait and Posture, vol. 27, pp. 455-462, 2008.

5. B. R. Umberger, "Effects of suppressing arm swing on kinematics, kinetics, and energetics of human walking," Journal of Biomechanics, vol. 41, pp. 2575-2580, 2008.

6. A. V. Hill, "The heat of shortening and the dynamic constants of muscle," Royal Society of London Proceedings Series B, vol. 126, pp. 136-195, 1938.

7. J. Doke and A. D. Kuo, "Energetic cost of producing cyclic muscle force, rather than work, to swing the human leg," The journal of experimental biology, vol. 210, pp. 2390-2398, 2007.

8. C. Vaughan, Dynamics of human gait. Kiboho Publishers, 1999.

9. A. M. Formal'skii, "Motion of anthropomorphic biped under implusive control," in Proc. of Institute of Mechanics, Moscow State Lomonosov University: "Some Questions of Robot's Mechanics and Biomechnics", 1978, (In Russian), pp. 17-34.

10. A. Formal'skii, Locomotion of Anthropomorphic Mechanisms. [In Russian], Nauka, Moscow, Russia, 1982.

11. A. M. Formal'skii, "Ballistic walking design via impulsive control," ASCE, Journal of Aerospace Engineering, vol. 23, no. 2, pp. 129-138, 2010.

12. S. Mochon and T. McMahon, "Ballistic walking: An improved model," Mathematical Biosciences, vol. 52, pp. 241-260, 1981.

13. T. McGeer, "Passive dynamic walking," International Journal of Robotics Research, vol. 9, no. 2, pp. $62-82,1990$.

14. Y. Hurmuzlu and T.-H. Chang, "Rigid body collisions of a special class of planar kinematic chains," IEEE Transactions on systems, man and cybernetics, vol. 22, no. 5, pp. 964-971, 1992.

15. P. H. Channon, S. H. Hopkins, and D. T. Pham, "Derivation of optimal walking motions for a bipedal walking robot," Robotica, vol. 10, no. 3, pp. 165-172, 1992.

16. C. Chevallereau and Y. Aoustin, "Optimal reference trajectories for walking and running of a biped," Robotica, vol. 19, no. 5, pp. 557-569, 2001.

17. P. Gill, W. Murray, and M. Wright, Practical optimization. London: Academic Press, 1981. 
18. M. Powell, Variable metric methods for constrained optimization, ser. Lecture Notes in Mathematics. Springer Berlin / Heidelberg, 1977, pp. 62-72.

19. J. Yoon, B. Novandy, C. H. Yoon, and K. J. Park, "A 6-dof gait rehabilitation robot with upper and lower limb connections that allows walking velocity updates on various terrains," IEEE/ASME Transactions on Mechatronics, vol. 15, no. 2, pp. 201-215, 2010.

20. T. Wannier, C. Bastiaanse, G. Colombo, and V. Dietz, "Arm to leg coordination in humans during walking, creeping and swimming activities," Experimental brain research, vol. 141, no. 2, pp. 375-379, 2001

21. Y. Aoustin and A. M. Formal'sky, "On optimal swinging of the biped arms," in Proc. IEEE Int. Conf. on Intelligent Robots and Systems IROS, Nice, France, 2008, pp. 2922-2927.

22. S. T. Eke-Okoro, M. Gregoric, and L. E. Larson, "Alterations in gait resulting from deliberate changes of arm-swing amplitude and phase," Clinical Biomechanics, vol. 12, no. $7 / 8$, pp. 516-521, 1997. 